\title{
Autoeficacia, Catastrofismo, Miedo al Movimiento y resultados de salud en la Fibromialgia
}

\author{
Manuel Tirado Zafra-Polo ${ }^{1 *}$, María A. Pastor-Mira ${ }^{2}$ y Sofía López-Roig ${ }^{2}$ \\ ${ }^{1}$ Universidad Cardenal Herrera CEU \\ ${ }^{2}$ Universidad Miguel Hernández.
}

\begin{abstract}
Resumen: Objetivo: Estudiar las relaciones y capacidad predictiva de la Autoeficacia, el Catastrofismo y el Miedo al Movimiento, sobre la experiencia de dolor en la Fibromialgia, además de identificar el papel de la Autoeficacia en el conjunto de estas relaciones. Método: Sesenta y un pacientes de la Unidad de Atención Integral a la Fibromialgia (Hospital San Vicente del Raspeig) cumplimentaron diferentes cuestionarios para evaluar las variables objeto de este estudio. Se utilizó el análisis de correlación de Pearson y el análisis de regresión múltiple. Resultados: No se encontraron relaciones significativas entre el Miedo al Movimiento y el resto de variables; solo la percepción del impacto global de la fibromialgia y del distrés presentó relaciones significativas tanto con el Catastrofismo como con la Autoeficacia. Esta última, no moduló la relación entre Catastrofismo y los resultados de salud. Sin embargo, además de su efecto directo en los mismos, mostró un efecto mediado por el Catastrofismo. Conclusión: La Autoeficacia aparece como la variable más relevante en la explicación de la experiencia de dolor de estos pacientes, constituyendo un objetivo terapéutico imprescindible también en el último nivel asistencial.

Palabras clave: Fibromialgia; autoeficacia; catastrofismo; miedo al movimiento; resultados de salud.
\end{abstract}

\section{Introducción}

La Fibromialgia es un síndrome de dolor crónico musculoesquelético caracterizado por fatiga, dolor generalizado y persistente, y rigidez de intensidad variable en los músculos, tendones y tejidos blandos, que se puede acompañar de otros problemas tanto físicos (jaquecas, parestesias o insomnio, entre otros) como psicológicos (dificultades de atención y memoria, estrés, ansiedad y depresión) (Bayazit, Gürsoy, Özer, Karakurum y Madenci, 2002). Su prevalencia está en torno al 2-3\% de la población y es diagnosticada con mayor frecuencia en mujeres (Carbonell-Abelló, 2005; Carmona et al., 2001). La fibromialgia también se caracteriza por producir una importante discapacidad y por la eficacia limitada de los tratamientos (Alegre de Miquel, Alejandra-Pereda, Betina-Nishishinya y Rivera, 2005). Actualmente, es la patología de partes blandas que genera más consultas en España (Gamero, Gabriel, Carbonell, Tornero y Sánchez-Magro, 2005; Pastor, Lledó, López, Pons y Martín-Aragón, 2010) suponiendo, además, un elevado coste sanitario. En atención primaria, la fibromialgia se asocia a un $61 \%$ de más gasto económico en relación con la ansiedad generalizada (SicrasMainar, Blanca-Tamayo, Navarro-Artieda y Rejas-Gutiérrez, 2009). Según algunos autores, el coste de un paciente con fibromialgia es de casi 10.000 euros al año, tres veces más que un paciente medio y doce veces más que un sujeto sano, de-

* Dirección para correspondencia [Correspondence address]: Manuel Tirado Zafra-Polo. C/ Carmelitas 3, 03203, Elche (Alicante, España). E-mail: manuel.tirado@uch.ceu.es
Title: Self-efficacy, Catastrophizing, Fear of Movement and health outcomes in Fibromyalgia.

Abstract: Objective: The aims of this research are to study the relationships and the predictive power of Self-Efficacy, Catastrophizing and Fear of Movement on the pain experience in Fibromyalgia, and to identify the role of self-efficacy in these relationships. Method: sixty one patients from the Fibromyalgia Integral Care Unity (San Vicente del Raspeig Hospital) completed several questionnaires for assessing the variables of this study. Data were analyzed using Pearson correlation and different multiple regression analysis were performed. Results: No significant relationships were found between fear of movement and other variables; only the perception of the overall impact of fibromyalgia and distress showed significant relationships with both Catastrophizing and Self-Efficacy. This last variable did not modulate the relationship between Catastrophizing and health outcomes. However, in addition to its direct effect over health outcomes, SelfEfficacy showed an effect mediated by the Catastrophizing. Conclusions: Self-Efficacy emerges as the most important variable in explaining the experience of pain in these patients, constituting a therapeutic target also in the last essential level of care.

Key words: Fibromyalgia; self-efficacy; catastrophizing; fear of movement; chronic pain; health outcomes.

bido, sobre todo, a la pérdida de jornadas laborales y a la incapacidad laboral (Rivera, Rejas, Esteve-Vives y Vallejo, 2009). Por tanto, nos encontramos ante un importante problema sociosanitario con numerosas consecuencias individuales, laborales y sociales.

La evidencia disponible muestra que la combinación de tratamiento farmacológico, psicológico y ejercicio físico resulta ser la pauta más eficaz para la mejoría de estos pacientes (Gombliewsky et al., 2010; Häuser, Bernardy, Arnold, Ofenbächer y Schiltenwolf, 2009). Glombiewski et al. (2010), en una revisión de la literatura, determinan que el tamaño del efecto de los cambios producidos por terapias cognitivo-conductuales es equivalente al obtenido con terapias farmacológicas, con la diferencia de que el tratamiento psicológico los mantiene estables, al menos durante dos años después de la intervención. Los tratamientos multidisciplinares con una orientación cognitivo-conductual, provocan cambios significativos relacionados con la percepción de control del dolor, la reducción de su intensidad, la interferencia del problema en la vida cotidiana y las mejoras emocionales (Thieme, Turk, y Flor, 2007). Algunos autores han mostrado cómo la percepción de autoeficacia en el manejo del dolor crónico es el proceso psicológico responsable de la mejoría obtenida en este tipo de tratamientos (Dobkin et al., 2010). Otros, han expresado la necesidad de considerarla junto con el Catastrofismo y el Miedo al Movimiento, a la hora de establecer grupos de tratamiento psicológico, dada la capacidad predictiva que los tres constructos han mostrado sobre el dolor y la discapacidad (Denison, Asenlöf, Sand- 
borgh y Lindberg, 2007). En cualquier caso, además de la relevancia de estos constructos en relación con los cambios producidos por los tratamientos cognitivo-conductuales, también es cierto que, de forma consistente, la literatura revisada muestra su importancia en los resultados de salud y en los procesos de adaptación al dolor crónico en general y a la fibromialgia, en particular (Pastor, Lledó, Pons y LópezRoig, 2011).

Las expectativas de autoeficacia hacen referencia a la creencia en las propias capacidades para organizar y ejecutar los cursos de acción necesarios para manejar situaciones futuras; se refiere, por tanto, a los juicios de cada persona sobre las capacidades que posee, y a partir de los cuales se comportará para poder alcanzar el rendimiento deseado (Bandura, 1982, 1997). En dolor crónico, las expectativas de autoeficacia se refieren a las creencias en la capacidad para manejar los síntomas diferentes del dolor, el funcionamiento físico y la misma percepción de dolor, en términos de intensidad (Anderson, Dowds, Pelletz, Edwards y PeetersAsdourian, 1995). En este contexto, esa percepción de autoeficacia, se ha asociado a un mejor estado de salud tanto en el caso del dolor crónico musculoesquelético (Gatchel et al., 2007; Pastor, Martín-Aragón y Lledó, 1999; Spinhoven et al., 2004) como en la Fibromialgia (González et al., 2009; Lledó et al., 2010; Pastor et al., 2011; Sharma et al., 2003). Además, también ha mostrado ser un proceso relevante en la predicción longitudinal del estado de salud de las pacientes con fibromialgia en el contexto de Atención Primaria (Pons, 2010) y en el uso de recursos sanitarios por parte de estos pacientes (Pastor et al., 2010). Por tanto, podemos afirmar que, según la literatura revisada, está bien establecido el efecto beneficioso de la Autoeficacia en el manejo del dolor tanto en su percepción, como en la discapacidad y el estado emocional, incluido el distrés, de las pacientes con fibromialgia. Sin embargo, cabe destacar que la mayoría de los estudios revisados, no han utilizado muestras de pacientes atendidas en el nivel terciario de salud, que representan las Unidades especializadas de Fibromialgia, ni han investigado su papel de forma simultánea con otras variables relevantes en la experiencia de dolor, como son el Catastrofismo y el Miedo al Movimiento (Pastor et al., 2011).

El Catastrofismo es otro de los constructos psicológicos importantes en los resultados de salud de la Fibromialgia y constituye un proceso clave en la manifestación de sus síntomas clínicos (García-Campayo et al., 2009). El Catastrofismo se entiende como un procesamiento cognitivo basado en pensamientos y expectativas negativas ante el dolor (Sullivan et al., 2001). Este tipo de procesamiento se centra exclusivamente en el dolor e incluye: la visión negativa y desproporcionada sobre el mismo (magnificación), la imposibilidad de parar de pensar en él (rumiación) y la imposibilidad de controlarlo (indefensión). Vlaeyen et al. (2004) encontraron que los pensamientos catastrofistas en relación con el dolor, se asociaban con un aumento de miedo en pacientes que iban a ser intervenidos de cardioversión eléctrica. Del mismo modo, se ha demostrado que las personas con un al- to catastrofismo, tienen más dificultades para desviar la atención de las señales dolorosas, debido a la errónea convicción de que estas señales anticipan un dolor más intenso (Van Damme et al., 2002). Otros autores, también han encontrado relaciones significativas entre el Catastrofismo y la reacción de ansiedad ante el dolor (Buenaver, Edwards y Haythornthwa, 2007), así como con la percepción de intensidad del dolor (Esteve, Ramírez-Maestre y Lopez-Martínez, 2007; Ramírez-Maestre, Esteve-Zarazoga, y López-Martínez, 2001). En el caso concreto de la Fibromialgia, el Catastrofismo se ha relacionado con mayor intensidad y sensibilidad al dolor y mayor discapacidad y depresión (Buenaver et al., 2008; Vowles, García-Campayo et al., 2008; McCracken, y Eccleston, 2007). Así pues, los trabajos revisados, muestran de forma consistente el efecto negativo del pensamiento catastrofista en los resultados de salud de los pacientes con dolor crónico y con fibromialgia.

En relación con el Miedo al Movimiento, existe acuerdo en considerarlo como un factor psicológico relevante en los procesos de cronificación del dolor, como, por ejemplo, en el caso del dolor lumbar (Leeuw et al., 2007). Además, diferentes trabajos han mostrado su efecto negativo en la experiencia de dolor crónico en general (Hasset et al., 2000; Martin et al., 1996; Turk et al., 2004) y en la discapacidad asociada (Boersma y Linton, 2005; Denison et al., 2004; Goubert et al., 2005; Peters et al., 2005). La creencia de que la actividad produce más dolor o de que, junto con el dolor, produce más daño, suele conducir a un patrón conductual caracterizado por el excesivo reposo y por la evitación del movimiento, que es altamente perjudicial en los procesos de dolor crónico (Peters, Vlaeyen y Weber, 2005). En el caso concreto de la Fibromialgia, existen pocos trabajos en la literatura revisada que se ocupen del miedo al movimiento y de sus relaciones con la experiencia de dolor de estas pacientes (Pastor et al., 2011). Sin embargo, cabe destacar que los resultados obtenidos son semejantes a los encontrados en otros problemas de dolor crónico; esto es, el Miedo al Movimiento se ha asociado con mayor discapacidad, emociones negativas e intensidad de dolor (De Gier, Peters y Vlaeyen, 2003; Turk et al., 2004).

A pesar de la importancia de cada uno de los constructos mencionados en la experiencia de dolor crónico de la Fibromialgia, no encontramos trabajos en la literatura revisada que los estudien de forma conjunta. Los resultados obtenidos hasta el momento, muestran la contribución independiente de la Autoeficacia, el Catastrofismo y el Miedo al Movimiento tanto en la percepción del dolor, como en la del estado de salud en general, la discapacidad y el estado emocional, valorado de diferentes formas. Sin embargo, hasta el momento, no se ha comprobado si esa contribución se ve modificada cuando se consideran de forma conjunta. Así pues, este trabajo tiene como objetivo estudiar las relaciones y capacidad predictiva de la Autoeficacia, el Catastrofismo y el Miedo al Movimiento, sobre la experiencia de dolor de la Fibromialgia. Además, pretende identificar el papel de la Autoeficacia en el conjunto de estas relaciones. Asumiendo 
la relación directa de la Autoeficacia en los resultados de salud de la Fibromialgia, ya mostrada en la literatura revisada, nos interesa profundizar en otros dos tipos de relación dado el papel fundamental que la Teoría Social Cognitiva atribuye a estas expectativas en el funcionamiento humano (Bandura, 1997): por un lado, explorar su posible efecto modulador en la relación del Catastrofismo y del Miedo al Movimiento con los resultados de salud, asumiendo como hipótesis de partida que el efecto negativo descrito para ambos constructos se produzca cuando la percepción de autoeficacia en el manejo del dolor sea baja; por otro, siguiendo una de las propuestas de la Teoría de la Autoeficacia (Bandura, 1977, 1982), que afirma su efecto no solo a través de la elección de actividades y conductas y de la persistencia y esfuerzo que se ponga en ellas, sino también a través de su influencia en los pensamientos y reacciones emocionales, nos interesa explorar su efecto en el estado de salud de las pacientes con Fibromialgia, mediado, entonces, por el Catastrofismo y el Miedo al Movimiento.

\section{Método}

\section{Participantes}

Participaron sesenta y un pacientes de la Unidad de Atención Integral a la Fibromialgia (UAI-FM) del Hospital de San Vicente del Raspeig (Alicante), con diagnóstico confirmado de Fibromialgia. Los criterios de inclusión fueron: personas mayores de 18 años, con diagnóstico de Fibromialgia según criterios ARA (Wolfe et al., 1990), que supieran leer y que acudieran a los talleres de tratamiento de la UAIFM. Los criterios de exclusión fueron: personas con problemas cognitivos o psiquiátricos y con problemas con el idioma que impidieran cumplimentar los cuestionarios por sí mismas.

\section{Procedimiento}

Los pacientes que, durante el período de abril a junio de 2011, participaron en alguna sesión del programa de 20 talleres que integran la intervención diseñada en la Unidad, recibieron por parte del psicólogo conductor de la investigación, un cuadernillo con los cuestionarios seleccionados. De los contactados en este periodo temporal $\quad(n=250)$, aceptaron participar 180 pacientes (72\%), pero solo 61 (34\%) entregaron en el plazo previsto (próxima visita a la unidad, dos o tres semanas más tarde). El procedimiento de captación fue diseñado para no interferir en el funcionamiento normal de la Unidad, además de no molestar a los pacientes añadiendo más sesiones a las que asistir y, por tanto, tener que desplazarse a la UAI-FM. Cabe señalar que esta Unidad es de referencia para toda la Comunidad Valenciana. Por ello, y dado que nos encontrábamos con sujetos entrenados en la realización de cuestionarios (por la propia dinámica de la Unidad), al finalizar cada taller, el psicólogo informaba a los pacientes de la investigación que se estaba realizando y leía la información del estudio al grupo. A continuación, cada persona firmaba un consentimiento informado y se entregaba el cuadernillo de cuestionarios para que los cumplimentaran en casa. De este modo, se intentaba evitar el posible efecto de recencia de los conocimientos aprendidos en el taller que acababan de recibir. Se les subrayaba la importancia de realizar este cuestionario de manera autónoma y con total sinceridad. Además, se les comunicaba que, en el caso de dudas, cuando devolvieran el cuestionario accederían al investigador para consultarlas. Los pacientes devolvieron los cuestionarios en la siguiente sesión a la que acudieron a la Unidad. Este estudio fue sometido a la aprobación del comité de ética del hospital.

\section{Variables e instrumentos}

En la Tabla 1 se presenta un resumen de las variables e instrumentos utilizados, así como los índices de consistencia interna de las diferentes escalas en la muestra de este estudio.

\section{Variables de Estado de Salud}

Itensidad de dolor: Valorada con la puntuación media en una escala de dolor diseñada ad hoc, basada en las propuestas de Jensen, Turner, Turner y Romano (1996), en la que se le pide al paciente que indique un número entre 0 y $10(0=$ "nada de dolor" y $10=$ "el peor dolor que pueda imaginar") para puntuar el mayor y menor dolor y el dolor general de la semana anterior, así como el dolor actual. Puntuaciones altas indican mayor intensidad de dolor, siendo el mínimo 0 puntos y el máximo 40 puntos. Jensen et al (1996) sugirieron como mejor medida en estudios clínicos la puntuación media del dolor actual y del menor dolor de la semana anterior. Sin embargo, en trabajos previos realizados con muestra española de pacientes con Fibromialgia, los datos psicométricos han apoyado el uso de los cuatro ítems de la escala como puntuación total de intensidad de dolor (Martín-Aragón et al., 1999; Lledó, Pastor, Pons, López-Roig y Terol, 2009). Por ello, en este trabajo se decidió utilizar la puntuación mencionada.

Impacto de la enfermedad y Discapacidad: Utilizamos la versión española de consenso del Fibromyalgia Impact Questionaire (FIQ) (Esteve-Vives, Rivera, Salvat, de Gracia y Alegre de Miquel, 2007). Es un cuestionario autoadministrado de 10 ítems que valora el estado de salud percibido de estos pacientes. El primer ítem se centra en la capacidad para realizar actividades físicas y puede utilizarse de manera aislada para evaluar el grado de discapacidad del paciente. En los siguientes se pregunta por el trabajo, dolor y otros síntomas de la fibromialgia. La escala de respuesta es analógica visual, entre 0 (mínimo impacto de la enfermedad) y 100 (máximo impacto). Es un instrumento recomendado por la Sociedad Española de Reumatología (SER) para evaluar tanto la discapacidad como el impacto global de la fibromialgia (Rivera et al., 2006). 
Distrés, Ansiedad y Depresión: Adaptación española de la Hospital Anxiety and Depression Scale (HAD) (Herrero et al., 2003). Escala autoadministrada de 14 ítems con respuestas tipo Likert de 0 a 3 puntos. También está recomendada por la SER como instrumento de elección para valorar el estado emocional de pacientes con fibromialgia (Rivera et al, 2006). Permite obtener una puntuación total de Distrés (sumatorio de todos los ítems) así como otra de Ansiedad y Depresión (sumatorio de los 7 ítems que componen cada subescala). A mayor puntuación mayor distrés, ansiedad o depresión, según el caso. Si bien en la mayoría de trabajos se utilizan las puntuaciones de Ansiedad y Depresión, decidimos incorporar la puntuación total como indicador de Distrés dado el carácter exploratorio del trabajo.

\section{Variables cognitivas}

Catastrofismo: Adaptación española del Pain Catastrophizing Scale (PCS) de García Campayo et al. (2008). Este cuestionario evalúa los pensamientos catastrofistas del paciente e incluye puntuaciones para las dimensiones de rumiación, magnificación e indefensión. Se trata de un cuestionario con 13 ítems con una escala de respuesta tipo Likert de cinco puntos ( $0=$ Nada en absoluto; $4=$ Todo el tiempo). Para este trabajo utilizamos únicamente la puntuación total. Ma- yores puntuaciones indican mayor presencia de pensamientos catastrofistas.

Miedo al Movimiento: Hemos utilizado la versión, recientemente validada al castellano, de la Tampa Scale for Kinesiopbobia (TSK) (Gómez-Pérez et al., 2010). Se trata de un cuestionario autoaplicado de 11 ítems acerca del miedo al movimiento o a la lesión. Los pacientes tienen que señalar su grado de acuerdo con cada una de la afirmaciones presentadas, utilizando una escala tipo Likert desde 1 (totalmente desacuerdo) hasta 4 (totalmente de acuerdo). Este cuestionario está diseñado para evaluar también las dimensiones de evitación de la actividad y daño, pero, en este trabajo, utilizaremos únicamente la puntuación total. Puntuaciones altas indican mayor miedo al movimiento.

Autoeficacia: Valorada con el Cuestionario de Autoeficacia en Dolor Crónico (Martín-Aragón et al., 1999). Utilizamos sólo la puntuación total, obtenida del sumatorio de las respuestas a los 19 ítems que integran el cuestionario. El sujeto debe contestar el grado en que se considera capaz de realizar determinadas actividades o de manejar su dolor, sus problemas emocionales u otros síntomas asociados al dolor crónico. Se contesta con una escala de respuesta tipo Likert de 0 (me veo totalmente incapaz) a 10 (me veo totalmente capaz). Puntuaciones altas indican alta percepción de autoeficacia.

Tabla 1. Variables, instrumentos e índices de consistencia interna en la muestra.

\begin{tabular}{|c|c|c|c|c|}
\hline & Instrumento & Variables & (rango teórico) & Alfa \\
\hline \multirow[t]{6}{*}{ Estado de salud } & Escala ad hoc & Intensidad Dolor & $(0-10)$ & .87 \\
\hline & FIQ & Impacto de la fibromialgia & $(0-100)$ & .80 \\
\hline & & Discapacidad & $(0-10)$ & .80 \\
\hline & HAD & Distrés & $(0-42)$ & .69 \\
\hline & & Ansiedad & $(0-21)$ & .67 \\
\hline & & Depresión & $(0-21)$ & .80 \\
\hline \multirow[t]{3}{*}{ Variables cognitivas } & PCS & Catastrofismo & $(0-52)$ & .92 \\
\hline & TSK & Miedo al Movimiento & $(11-44)$ & .78 \\
\hline & CADC & Autoeficacia & $(0-190)$ & .94 \\
\hline
\end{tabular}

FIQ: Fybromialgia Impact Questionnaire; HAD: Hospital Anxiety and Depression; PCS: Pain Catastrophizing Scale; TSK: Tampa Scale of Kinesiophobia; CADC: Cuestionario de Autoeficacia en Dolor Crónico

\section{Análisis estadístico}

Utilizamos el programa SPSS 19.0. Se realizó un estudio descriptivo para establecer las características de la muestra y de las variables objeto de este trabajo. Utilizamos el análisis de correlación de Pearson para estudiar las relaciones entre las variables, seleccionando para los posteriores análisis de regresión las variables cognitivas que presentaron relaciones significativas con las de estado de salud. Dado el número de relaciones, utilizamos como criterio un nivel de significación $p \leq .01$, pues la corrección de Bonferroni es demasiado estricta y puede incrementar la probabilidad de error tipo II.

Para establecer los modelos predictivos y estudiar el posible efecto modulador de la Autoeficacia en la relación entre catastrofismo y miedo al dolor con los resultados de salud, realizamos diferentes análisis de regresión jerárquicos donde en una primera fase introdujimos (ENTER) el mode- lo máximo, combinándolo con una eliminación por pasos (BACKWARD) de los términos de interacción. Como resultado de lo anterior, establecimos un nuevo modelo máximo cuyas variables se volvieron a introducir combinando, de nuevo, con la eliminación por pasos y manteniendo el principio jerárquico. En todos los casos se comprobaron los requisitos de ausencia de colinealidad (Índice de condición < 30) y de independencia (Durbin-Watson entre 1.5-2.5).

Para estudiar el posible efecto mediador del catastrofismo y el miedo al movimiento en las relaciones establecidas entre la autoeficacia y los resultados de salud, seguimos el procedimiento de Baron y Kenny (1986) que implica la realización de tres análisis de regresión y el cumplimiento de cuatro requisitos. En el primer análisis se introduce la variable independiente para predecir la mediadora; en el segundo, la variable independiente para predecir la variable resultado y, en el tercero, se introduce simultáneamente la variable in- 
dependiente y la mediadora para predecir la variable resultado. La mediadora funciona como tal cuando: 1) la variable independiente es un predictor significativo de la mediadora; 2) la variable independiente es un predictor significativo de la variable resultado; 3) la variable mediadora es un predictor significativo de la variable resultado y 4) el efecto de la variable independiente en la variable resultado es menor en la tercera ecuación que en la segunda. Para determinar si ese descenso es significativo no pudimos utilizar el Test de Sobel (1982) debido a su exigencia con el tamaño muestral mínimo de 125 sujetos (Fritz y MacKinnon, 2007); por tanto hemos seguido exclusivamente las indicaciones arriba mencionadas.

\section{Resultados}

\section{Descripción de la Muestra}

De los 61 participantes en el estudio, 58 eran mujeres y 3 hombres, con edades comprendidas entre los 26 y 75 años $(M=50.5 ; D T=11.5$; Mujeres: $51 ; D T=11.4$; Hombres: 42.2; $D T=12.6)$. La mayoría se sitúa en los niveles de estudio primarios y medios, está casada y trabajando en el momento de la recogida de datos (Tabla 2).

Durante el periodo de evaluación, solo 6 pacientes no se encontraban consumiendo medicación. En el tiempo que llevaban asistiendo a la UAI-FM $(M=0.75$ años; $D T=1.10$; Rango $=0.07-6.26)$ cada paciente ha sido atendido médicamente una media de 1.52 veces $(D T=1.01$; Rango $=1-7) \mathrm{y}$ psicológicamente una media de 0.39 veces $(D T=.76$; Rango = 0-3); además, ha acudido como media a 8.15 talleres sobre un total de $20(D T=4.43$; Rango $=2-20)$.

Tabla 2. Características sociodemográficas

\begin{tabular}{lcc}
\hline & Frecuencia & Porcentaje $\%$ \\
\hline Nivel de estudios & 3 & \\
Lee/escribe & 29 & 4.9 \\
Estudios primarios & 24 & 47.5 \\
Estudios medios & 25 & 39.3 \\
Estudios universitarios & & 8.2 \\
Estado civil & 6 & \\
Soltero & 40 & 9.8 \\
Casado & 11 & 65.6 \\
Divorciado & 4 & 18.0 \\
Viudo & & 6.6 \\
Situación laboral & 27 & \\
Trabajando & 2 & 44.3 \\
Paro & 21 & 3.3 \\
Ama de casa & 11 & 34.4 \\
Jubilado & 0 & 18.0 \\
Incapacidad laboral & & -- \\
\hline
\end{tabular}

Descripción de las variables objeto de estudio y análisis de relaciones

Como se puede observar en la Tabla 3, las puntuaciones de la mayoría de las variables se sitúan en valores medios, considerando el rango teórico expuesto en la Tabla 1.
Tabla 3. Análisis descriptivo.

\begin{tabular}{llll}
\hline Variable & Media & DT & Rango \\
\hline Intensidad de dolor & 6.4 & 1.7 & $2.25-10$ \\
Discapacidad & 4.89 & 1.95 & $0.67-9.66$ \\
Impacto Fibromialgia & 64.58 & 15.19 & $34.20-97.68$ \\
Distrés & 22.21 & 6.04 & $8-37$ \\
Ansiedad & 12.52 & 3.93 & $3-20$ \\
Depresión & 9.69 & 3.42 & $2-18$ \\
Catastrofismo & 28.82 & 10.91 & $2-45$ \\
Miedo Movimiento & 29.67 & 7.03 & $15-44$ \\
Autoeficacia & 92.36 & 38.26 & $14-185$ \\
\hline
\end{tabular}

Los resultados del análisis de correlación muestran a la Autoeficacia como la variable que establece un mayor número de relaciones significativas, y en el sentido esperado desde la teoría, con las variables de estado de salud evaluadas ( 5 de un total de 6) con valores que oscilan entre $r=-.38$ en el caso de la intensidad del dolor y $r=-.54$ en el caso de la depresión (Tabla 4). La ansiedad fue la única variable resultado que no presentó relaciones significativas con la Autoeficacia.

Tabla 4. Análisis de correlación

\begin{tabular}{|c|c|c|c|c|c|c|c|c|}
\hline$\overline{\text { VARIABLES }}$ & 1 & 2 & 3 & 4 & 5 & 6 & 7 & 8 \\
\hline \multicolumn{9}{|l|}{ 1.Distrés } \\
\hline 2.Ansiedad & .85 & & & & & & & \\
\hline 3.Depresión & .80 & .35 & & & & & & \\
\hline 4.Impacto FM & .43 & .32 & .40 & & & & & \\
\hline 5.Discapacidad & .20 & .01 & .35 & .55 & & & & \\
\hline $\begin{array}{l}\text { 6.Intensidad do- } \\
\text { lor }\end{array}$ & .07 & .05 & .06 & .60 & .44 & & & \\
\hline 7.Catastrofismo & .42 & .41 & .28 & .43 & .23 & .25 & & \\
\hline $\begin{array}{l}\text { 8.Miedo al mo- } \\
\text { vimiento }\end{array}$ & -.04 & .02 & -.09 & .14 & .01 & .24 & .09 & \\
\hline 9.Autoeficacia & -.46 & -.24 & -.54 & -.51 & -.53 & -.38 & -.42 & -.01 \\
\hline
\end{tabular}

Por su parte, el catastrofismo, presentó relaciones significativas de signo positivo con la puntuación total de distrés, la ansiedad y el impacto global de la fibromialgia, con valores de $r$ en torno a .40 en los tres casos (Tabla 4). Finalmente, cabe destacar la ausencia de relaciones significativas del Miedo al Movimiento con todas las variables de estudio.

\section{Análisis predictivo}

Solo la percepción del impacto global de la fibromialgia y del distrés presentó relaciones significativas con el Catastrofismo y la Autoeficacia. Así, realizamos los análisis de regresión considerando como variables criterio esos dos resultados de salud y como variables predictoras el Catastrofismo, la Autoeficacia y la interacción entre ambas. En el caso del impacto de la fibromialgia, dada su relación teórica (como problema primario de estos pacientes) y empírica ( $r=.60$. Tabla 4) con la intensidad del dolor, también consideramos esta variable en el análisis correspondiente. En la Tabla 5 se muestra el modelo final obtenido. 
Tabla 5. Análisis de regresión ${ }^{\mathrm{a}}$

\begin{tabular}{|c|c|c|c|c|}
\hline Criterio & Predictores & $F$ & $\beta$ & $R^{2} g l$ \\
\hline DISTRÉS & $\begin{array}{l}\text { Catatrofismo } \\
\text { Autoeficacia }\end{array}$ & $10.98^{* * *}$ & $\begin{array}{r}.28^{*} \\
-.34^{* *}\end{array}$ & .2560 \\
\hline IMPACTO FM & $\begin{array}{l}\text { Intensidad de dolor } \\
\text { Catastrofismo } \\
\text { Autoeficacia }\end{array}$ & $18.32^{* * *}$ & $\begin{array}{l}.45^{* * *} \\
.22^{*} \\
-.25^{*}\end{array}$ & .4660 \\
\hline
\end{tabular}

Como se puede observar, quedaron excluidos los términos de interacción en ambos casos, manteniéndose tanto el Catastrofismo como la Autoeficacia como predictores significativos de la percepción de distrés y, junto con la intensidad del dolor, del impacto global de la fibromialgia. En am- bos, la Autoeficacia tuvo una contribución más relevante que el Catastrofismo, considerando también al valor de las correlaciones semiparciales. Así, en relación con el Distrés, el Catastrofismo presentó un valor menor $(r=.25$; varianza explicada $=6.25 \%)$ que el obtenido con la Autoeficacia $(r=$ -.34; varianza explicada $=11.56 \%)$. Esta situación es semejante respecto de las correlaciones semiparciales obtenidas con la percepción del impacto de la Fibromialgia (Catastrofismo: $r=.19$; varianza explicada $=3.61 \%$; Autoeficacia: $r=$ -21 ; varianza explicada $=4.41 \%$ ) aunque, en este último caso, la intensidad del dolor tuvo la contribución más importante $(r=.41$; varianza explicada $=16.81 \%)$.

Respecto de la otra posible relación formulada para la Autoeficacia y los resultados de salud (efecto mediado, en este caso, sólo por los pensamientos catastrofistas sobre el dolor), en la Figura 1 se presentan los resultados obtenidos.

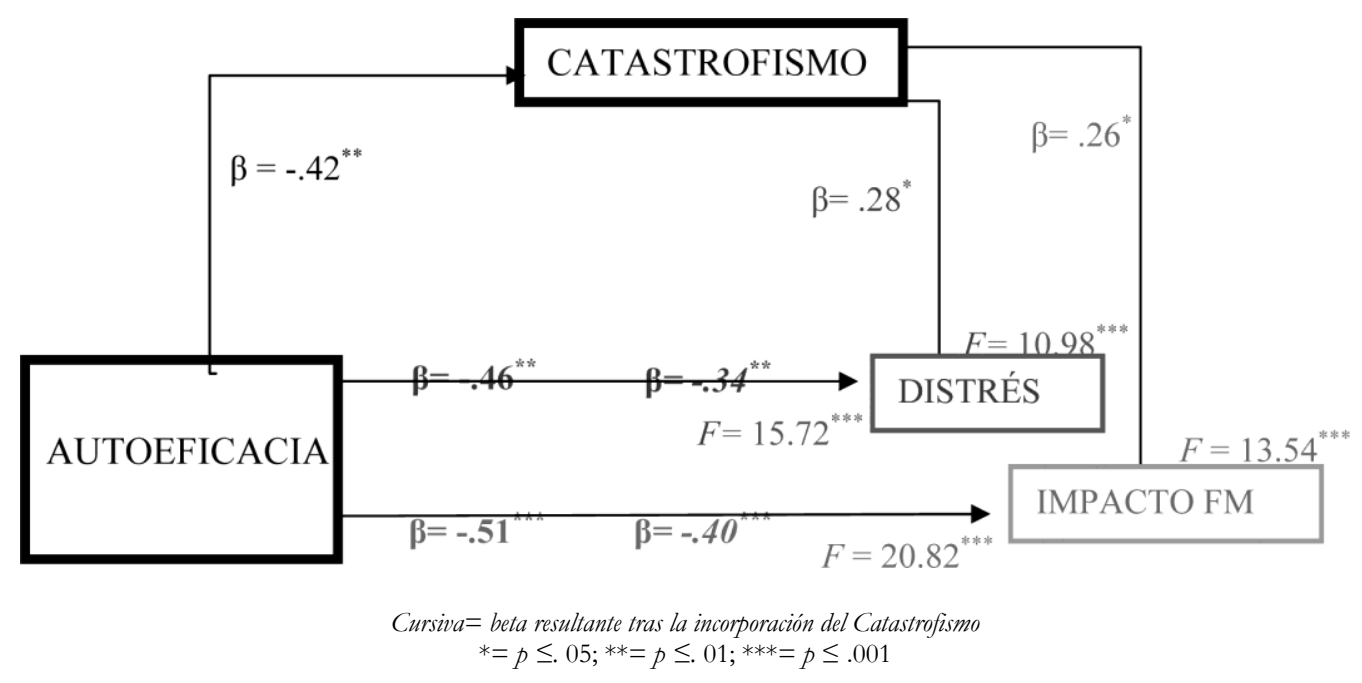

Figura 1. Análisis de mediación del Catastrofismo

Como puede observarse, el valor del coeficiente beta correspondiente a la Autoeficacia disminuye al introducir el Catastrofismo, tanto en el caso del distrés como en el del impacto global.

\section{Discusión}

El objetivo de este trabajo era explorar las relaciones de la Autoeficacia, el Catastrofismo y el Miedo al Movimiento con los resultados de salud de la fibromialgia, así como identificar el papel de la Autoeficacia en el conjunto de estas relaciones.

Antes de pasar a discutir los resultados obtenidos, cabe señalar que la consistencia interna de las diferentes escalas está por encima de .70, salvo en el caso del Distrés y la Ansiedad, próximas a ese valor. Considerando los criterios de Bisquerra (1989) todas ellas han presentado una consistencia interna adecuada en la muestra de estudio.
En general, los resultados respecto de los efectos positivos de la Autoeficacia en la experiencia de dolor de estos pacientes, son coincidentes con los de otros autores, tanto en relación con la intensidad del dolor como con el impacto general sobre el estado de salud y emocional del problema (Lledó et al., 2010; Vallejo et al., 2009; Velasco et al., 2008). De hecho, en este trabajo, la percepción de autoeficacia ha sido la única variable cognitiva que ha presentado relaciones significativas con la depresión, la discapacidad y la intensidad del dolor. Del mismo modo, las relaciones significativas obtenidas respecto del Catastrofismo, sugieren su importancia en la ansiedad informada por estos pacientes, siendo coherente con los resultados de otros estudios (Arnow et al., 2011; Börsbo, Gerdle y Peolsson, 2010; Gormsen, Rosenberg, Bach y Jensen, 2010; Jensen et al., 2010) y con las formulaciones teóricas acerca del papel de los pensamientos catastrofistas en el incremento de la ansiedad, tanto en el caso de estos pacientes como en el de otros problemas de dolor crónico (Leeuw et al., 2007; Pastor et al., 2011). La ausencia 
de relaciones significativas con la Depresión, apoyaría la interpretación anterior aunque este es un resultado específico de este trabajo pues los autores anteriores encontraron relaciones significativas también con esta variable (Brösbo et al., 2010; Gormsen et al., 2010; Jensen et al., 2010). Así pues, aunque tanto la Autoeficacia como el Catastrofismo se han relacionado con el distrés global en el sentido esperado desde la teoría, cabe destacar la relación diferencial que han establecido con la ansiedad y la depresión. Finalmente, otro de los resultados coincidentes con los obtenidos en otros contextos asistenciales, es la ausencia de relaciones significativas de la intensidad del dolor con el estado emocional (Lledó et al., 2010; Velasco et al., 2008) sugiriendo que ambos son dos problemas concurrentes en la FM, que pueden coexistir sin necesidad de relación entre ellos.

En este trabajo, también hemos obtenido resultados discrepantes con los de la literatura revisada. En primer lugar, la intensidad del dolor se ha relacionado significativamente con la discapacidad y con el impacto general percibido, en contra de la ausencia de relaciones informada en otros trabajos (Lledó et al., 2010; Vallejo et al., 2009; Velasco et al., 2008). Es posible que esta diferencia se deba a la situación de los pacientes en el contexto asistencial terciario, donde se derivan aquellas personas que informan de mayor gravedad en su problema de dolor. En segundo lugar, resulta llamativa la ausencia de relaciones significativas del Catastrofismo con la intensidad del dolor, siendo éste un resultado inesperado, por ser contrario tanto a la literatura revisada (Buenaver et al., 2008; Esteve et al., 2001; Leeuw et al., 2007; Pastor et al., 2011; Turk et al., 2004; Vowles, 2007) como al propio contenido del constructo. Aunque otros autores han encontrado esa misma ausencia de relaciones entre catastrofismo e intensidad de dolor (Gormsen et al., 2010), resulta difícil comparar ambos resultados debido a la utilización de instrumentos diferentes y a la gran discrepancia entre las muestras utilizadas. En el caso del estudio de Gormsen et al (2010) eran pacientes que no acudían a un centro de tratamiento, reclutados sin el control del seguimiento médico, sin tomar medicación o sin la exclusión de problemas psicológicos o psiquiátricos. En tercer y último lugar, otro de los resultados inesperados ha sido la ausencia de relaciones significativas del Miedo al Movimiento con las variables objeto de este estudio, a diferencia de lo obtenido en trabajos previos (Leeuw et al., 2007; Pastor et al., 2011; SwinkelsMeewisse, 2003). A pesar de lo sorprendente de este resultado, podría tener sentido en este contexto pues, curiosamente, ninguno de los pacientes estudiados se encontraba en situación de baja laboral. De hecho, la mayoría de la muestra estaba en activo, bien trabajando fuera de casa, bien como amas de casa. Es decir, la evitación de la actividad, que es parte integrante del constructo, no parece ser una experiencia presente en nuestra muestra, al menos en el momento de este estudio. En esta misma línea argumental, cabe señalar que la puntuación media obtenida en el Miedo al Movimiento por nuestros pacientes (29.7) es sensiblemente inferior a la obtenida por otros autores tanto con pacientes de fibro- mialgia como de otros problemas de dolor crónico (Denison et al., 2004; Gómez-Pérez et al., 2011; Goubert et al., 2005; Turk et al., 2004) donde oscila entre 32.71 y 39.00. En el mismo sentido, la puntuación media obtenida en la variable Discapacidad se situó en torno al 5 , en un rango teórico de 0 a 10.

En relación con el estudio del papel de la Autoeficacia en las relaciones encontradas, nuestros resultados descartan su efecto modulador en la relación entre Catastrofismo y distrés e impacto de la enfermedad. Sin embargo, muestran, por un lado, su mayor contribución directa en la explicación de estos resultados y, por otro, su contribución indirecta a través de la mediación de la forma de procesar la experiencia dolorosa (Catastrofismo). Este resultado coincide con el presupuesto de la teoría de la Autoeficacia (Bandura 1980, 1997) acerca de su influencia a través de los pensamientos y reacciones emocionales. En este caso, considerando, además, la relación de signo negativo entre Autoeficacia y Catastrofismo, podemos afirmar que las personas que se perciben autoeficaces en el manejo de su problema de dolor, procesan de forma menos catastrofista la situación dolorosa, con el consiguiente efecto beneficioso en las consecuencias de la enfermedad.

Este estudio tiene una serie de limitaciones a tener en cuenta. En primer lugar, es un estudio transversal y de naturaleza correlacional; diseños longitudinales son más adecuados para probar relaciones de mediación. En segundo lugar, al ser un estudio de campo realizado durante un determinado período de tiempo, hemos debido de adaptar el procedimiento de recogida de datos al funcionamiento de la unidad asistencial (a la que acudían pacientes de las tres provincias de la Comunidad Valenciana) y al período de estancia del psicólogo conductor del trabajo. Esta situación, ha hecho imposible seleccionar solo pacientes de nuevo ingreso, que hubiera evitado el posible efecto de aprendizaje por la participación en los talleres de la UAI-FM. Además, esto ha tenido consecuencias en el tamaño muestral conseguido, limitando el análisis a las puntuaciones totales de los constructos y no pudiendo utilizar sus diferentes dimensiones. Sin embargo, aunque el tamaño muestral resulta una limitación, la naturaleza exploratoria del estudio y la ausencia de asimetría en las variables utilizadas permite aportar información relevante para futuros estudios con objetivos inferenciales. Finalmente, y relacionado con lo anterior, a pesar de que cada vez hay más trabajos que analizan las diferencias entre hombres y mujeres, hemos optado por considerar la muestra como un todo y no reducir así su tamaño. Los pocos estudios disponibles sobre estas diferencias (Miró, Diener, Martínez, Sánchez y Valenza, 2012; Ruiz-Pérez et al, 2007) no están realizados en el nivel asistencial que nos ocupa y presentan resultados inconsistentes. El nivel terciario al que pertenece nuestra muestra, representa el último escalón al que pueden acceder los pacientes y llegan a él cuando el personal sanitario estima que la gravedad del problema es importante, por lo que necesitan soluciones con un alto grado de especialización. Esta situación podría reducir la variabilidad en la 
situación de salud de los pacientes; de hecho hombres y mujeres obtuvieron puntuaciones medias semejantes en el impacto percibido de la Fibromialgia evaluado con el FIQ (Mujeres: $M=64.8 ; D T=15.1$; Hombres: $M=61 ; D T=20.5)$ y en la percepción de intensidad de dolor (Mujeres: $M=6.4$; $D T=1.7$; Hombres: $M=6.6 ; D T=1.7)$. Cabe señalar que la distribución de nuestra muestra según el género refleja la distribución habitual de esta variable en Fibromialgia. Sin embargo, esto no parece ser el caso en relación con la situación laboral, donde resulta sorprendente la ausencia de bajas laborales. Este dato, junto con que hemos trabajado en el nivel asistencial terciario limita la validez externa del estudio. Se necesita, por tanto, replicar estos datos ampliando el tamaño muestral, planteando un estudio longitudinal y, además, profundizando en el estudio de las relaciones establecidas utilizando, también, las dimensiones componentes de los constructos de este estudio y del análisis diferencial considerando el género. Podría ser interesante, además, incorporar otros constructos relevantes en los resultados de salud de la fibromialgia como, por ejemplo, el afecto positivo y negativo (Hasset et al, 2008) o explorar la existencia de perfiles psicosociales diferentes según los resultados de la investigación en otros contextos asistenciales (Turk et al., 1996).

\section{Referencias}

Alegre de Miquel, C., Alejandra-Pereda, C., Betina-Nishishinya, M., y Rivera, J. (2005). Revisión sistemática de las intervenciones farmacológicas en la fibromialgia. Medicina clínica, 125, 784-787. doi: 10.1016/S00257753(05)72190-4

Anderson, K. O., Dowds, B. N., Pelletz R. E., Edwards, W. T., y PeetersAsdourian, C. (1995). Development and initial validation of a scale to measure self-efficacy beliefs in patients with chronic pain. Pain, 63(1), 77-84. doi: 10.1016/0304-3959(95)00021-J.

Arnow, B. A., Blasey, C. M., Constantino, M. J., Robinson, R., Hunkeler, E., Lee, J., Fireman, B., Khaylis, A., y Feiner, L. (2011). Catastrophizing, depression and pain-related disability. C. General Hospital Psychiatry, 33, 150-6.

Arnstein, P., Caudill, M., Mandle, C. L., Norris, A., y Beasley, R. (1999). Self efficacy as a mediator of the relationship between pain intensity, disability and depression in chronic pain patients. Pain, 80, 483-491.

Bandura, A. (1982). Self-efficacy mechanism in human agency. American Psychologist, 37, 122-147. doi: 10.1037/0003-066X.37.2.122

Bandura, A. (1997). Self-efficacy: The exercise of control. New York: Freeman.

Baron, R. M., y Kenny, D. A. (1986). The Moderator-Mediator Variable Distinction in Social Psychological Research: Conceptual, Strategic, and Statistical Considerations. Journal of Personality and Social Psychology, 51, 1173-1182. doi: 10.1037/0022-3514.51.6.1173

Bayazit, Y., Gürsoy, S., Özer, E., Karakurum, G., y Madenci, E. (2002). Neurotologic manifestations of fibromyalgic syndrome. Journal of Neurological Sciences, 196, 77-80.

Bisquerra, R. (1989). Introducción conceptual al análisis multivariante (Vol. II). Barcelona: PPU.

Boersma, K., y Linton, S. J. (2005). Screening to identify patients at risk: profiles of psychological risk factors for early intervention. Clinical Journal of Pain, 21, 38-43.

Börsbo, B., Gerdle, B., y Peolsson, M. (2010) Impact of the interaction between self-efficacy, symptoms and catastrophizing on disability, quality of life and health in chronic pain patients. Disability and Rebabilitation, 17, 1387-1396. doi:10.3109/09638280903419269

Brekke, M., Hjortdahl, P., y Kvien, T. K. (2001). Self-efficacy and health status in rheumatoid arthritis: a two-year longitudinal observational study. Rheumatology 40, 387-392. doi: 10.1093/rbeumatology/40.4.387
A pesar de las limitaciones mencionadas, este trabajo tiene el valor de haber puesto de manifiesto, en el último escalón asistencial representado por una Unidad especializada en Fibromialgia, el papel relevante de la Autoeficacia como predictor del estado de salud informado por los pacientes; de forma directa en todas las áreas de estado de salud evaluadas (salvo en la ansiedad) y de forma indirecta en el distrés e impacto global de la fibromialgia a través de la mediación del Catastrofismo. De nuevo, y también en este contexto asistencial, los resultados sugieren la potencialidad terapéutica de tener a la autoeficacia como objetivo de tratamiento. Parece claro que incrementar su percepción no sólo producirá efectos beneficiosos en diferentes áreas del estado de salud de las personas con fibromialgia, sino que también favorecerá un procesamiento menos catastrofista de la información dolorosa, lo cual, a su vez, tendrá efectos positivos tanto en el distrés como en el impacto global de la enfermedad.

Agradecimientos.- Este trabajo no hubiera podido realizarse sin la colaboración y ayuda del equipo de profesionales de la Unidad de Atención Integral a la Fibromialgia del Hospital de San Vicente del Raspeig, Alicante. Los autores también quieren agradecer la colaboración de todos los pacientes que aceptaron participar en el trabajo.

Buenaver, L. F., Edwards, R. R., y Haythornthwa, J. A. (2007). Pain-related catastrophizing and perceived social responses: Inter-relationships in the context of chronic pain. Pain, 127, 234-42. doi:10.1186/1745-6215$10-24$

Buenaver, L. F., Edwards, R. R., Smith, M. T., Gramling, S. E., y Haythornthwaite, J. A. (2008). Catastrophizing and pain-coping in young adults: Associations with depressive symptoms and headache pain. Journal of Pain, 9, 311-9. doi: 10.1016/j.jpain.2007.11.005

Carbonell-Avelló, J. (2005). Fibromialgia. Medicina clínica, 125, 778-9. doi: 10.1016/S0025-7753(05)72188-6

Carmona, L., Ballina, F. I., Gabriel, R., Laffon, A., y EPISER Study Group. (2001). The Burden of musculoskeletal diseases in the general population of Spain: results from national survey. Annals of the Rheumatic Diseases, 60, 1040-45.

De Andrés, J., Soriano, J. F., Monsalve, V,. y Soucase, B. (2004). Estrategias de afrontamiento ante el dolor y calidad de vida en pacientes diagnosticados de fibromialgia. Revista de la Sociedad Española del Dolor, 11, 353359.

De Gier, M., Peters, M. L., y Vlaeyen, J. W. (2003). Fear of pain, physical performance, and attentional processes in patients with fibromyalgia. Pain, 104, 121-30.

Denison, E., Asenlöf, P., Sandborgh, M., y Lindberg, P. (2007). Musculoskeletal pain in Primary Health Care: Subgroups based on pain intensity, disability, self-efficacy, and fear-avoidance variables. The Journal of Pain, $8,67-74$.

Denison, E., Asenlof, P., y Lindberg, P. (2004). Self-efficacy, fear avoidance, and pain intensity as predictors of disability in subacute and chronic musculoskeletal pain patients in primary health care. Pain, 111, 245-252. doi:10.1016/j.pain.2004.07.001

Dobkin, P. L., Liu, A., Abrahamowicz, M., Lonescu-Ittu, R., Sasha Bernatsky, S., Goldberger, A., y Baron, M. (2010). Predictors of Disability and Pain Six Months After the Endof T reatment for Fibromyalgia. Clinical Journal of Pain, 26, 23-29

Esteve, R., Ramirez-Maestre, C., y López-Martinez, A. E. (2007). Adjustment to chronic pain: the role of pain acceptance, coping strategies, and pain-related cognitions. Annals of Behavioral Medicine. 33, 179-88

Esteve-Vives, J., Rivera-Redondo, J., Salvat, M. I., de Gracia-Blanco, M., y Alegre de Miquel, C. (2007). Propuesta de una versión de consenso del 
Fibromyalgia Impact Questionnaire (FIQ) para la población española. Reumatología clínica, 21-24. Doi: 10.1016/S1699-258X(07)73594-5

Fritz, M. S. y MacKinnon, D. P. (2007). Required Sample Size to Detect the Mediated Effect. Psychological Science, 18, 233-239. Doi: 10.1111/j.14679280.2007.01882.x

Gamero, F., Gabriel, R., Carbonell, J., Tornero, J., y Sánchez-Magro, I. (2005). Pain in spanish Rheumatology outpatient offices: EPIDOR epidemiological study. Revista Clínica Española, 205, 157-163.

García-Campayo, J., Rodero, B., Alda, M., Sobradiel, N., Montero, J., y Moreno, S. (2008).Validación de la versión española de la escala de la catastrofización ante el dolor (Pain Catastrophizing Scale) en la fibromialgia. Medicina clínica, 131, 487-492.

García-Campayo, J., Serrano-Blanco, A., Rodero, B., Magallón, R., Alda, M., Andrés, E., Luciano, J. V., y López del Hoyo, Y. (2009). Effectiveness of the psychological and pharmacological treatment of catastrophization in patients with fibromyalgia: a randomized controlled trial. Trials, 10, 24-29. doi:10.1186/1745-6215-10-24

Gatchel, R. J., Peng, Y. B., Peters, M. L., Fuchs, P. N., y Turk, D. C. (2007). The biopsychosocial approach to chronic pain: Scientific advances and future directions. Psychological Bulletin, 133, 581-624 doi: 10.1037/00332909.133.4.581.

Glombiewski, J. A., Sawyer, A. T., Gutermann, J., Koenig, K., Rief, W. y Hofmann, S. G. (2010). Psychological treatments for fibromyalgia: A meta-analysis. Pain, 151, 280-288.

Gómez-Pérez, L. y López-Martínez, A. E. (2011). Psychometric Properties of the Spanish Version of the Tampa Scale for Kinesiophobia (TSK). Journal of Pain, 12, 425-435. Doi: 10.1016/j.jpain.2010.08.004

González, J. L., Peñacoba, C., Velasco, L., López, A., Mercado, F., y Barjola, P. (2009). Recursos cognitivos de percepción de control, procesos de estrés/ recuperación y malestar afectivo en fibromialgia. Psicothema, 21, 359-368.

Gormsen, L., Rosenberg, R., Bach F. W., y Jensen, T. S. (2010) Depression, anxiety, health-related quality of life and pain in patients with chronic fibromyalgia and neuropathic pain. European Journal of Pain, 14, 127-135.

Goubert, L., Crombez, G., y Van Damme, S. (2004). The role of neuroticism, pain catastrophizing and pain-related fear in vigilance to pain: a structural equations approach. Pain, 107, 234-241.

Hassett, A. L., Cone, J. D., Patella, S. J., y Sigal, L. H. (2000). The role of catastrophizing in the pain and depression of women with fibromyalgia syndrome. Arthritis and Rheumatism, 43, 2493-500. Doi: 10.1002/15290131(200011)43:11<2493::AID-ANR17>3.0.CO;2-W

Hasset A. L., Simonelli L. E., Radvanski D. C., Buyske S, Savage S. V., y Sigal L. H. (2008) The relationship between affect balance style and clinical outcomes in fibromyalgia. Arthritis and Rheumatism, 59, 833-40. Doi: $10.1002 /$ art. 23708

Häuser, W., Bernardy, K., Arnold, B., Offembächer, M., y Schiltenwolf, M. (2009). Efficacy of Multicomponent Treatment in Fibromyalgia Syndrome: A Meta-Analysis of Randomized Controlled Clinical Trials. Arthritis \& Rheumatism (Arthritis Care \& Research), 61, 216-224.

Herrero, M. J., Torres, X., Blanch, J., Peri, J. M., Pintor, L., y Bulbena, A. (2003). A validation study of the Hospital anxiety and depression scale (HADS) in a Spanish population. General Hospital Psychiatry, 25, 277-83. Doi: 10.1016/S0163-8343(03)00043-4)

Jensen, K. B., Petzke, F., Carville, S., Fransson, P., Marcus, H., Williams, S. C. R. Choy, E., Mainguy, Y., Gracely, R., Ingvar, M., y Kosek, E. (2010). Anxiety and Depressive Symptoms in Fibromyalgia are related to poor perception of health but not to pain sensitivity or cerebral processing of pain. Arthritis \& Rheumatism, 62, 3488-3495. doi: 10.1002/art.27649.

Jensen, M. P., Turner, L. R., Turner, J. A., y Romano, J. M. (1996). The use of multiple-item scales for pain intensity measurement in chronic pain patients. Pain, 67, 35-40.

Lacker, J. M., Carosella, A. M., y Feuerstein, M. (1996). Pain expectancies, pain, and functional self-efficacy expectancies as determinants of disability in patients with chronic low back disorders. Journal of Consult in Clinical Psychology, 64, 212-220.

Lackner, J. M. y Carosella, A. M. (1999). The relative influence of perceived pain control, anxiety, and functional self efficacy on spinal function among patients with chronic low back pain. Spine, 24, 2254-2261. doi: 10.1097/00007632-199911010-00014
Leeuw, M., Goossens, M. E. J. B., Linton, S. J., Crombez, G., Boersma, K. y Vlaeyen, J. W. S. (2006). The Fear-Avoidance Model of Musculoskeleta Pain: Current State of Scientific Evidence. Journal of Behavioral Medicine, 30, 77-94. doi: 10.1007/s10865-006-9085-0

Lledó, A., Pastor, M. A., Pons, N., López-Roig, S \& Terol, M.C. (2009). Comparing Fibromyalgia patients from Primary Care and Rheumatology settings: clinical and psychosocial features. Rheumatology International. Clinical and Experimental Investigations, 29, 1551-1160. doi: 10.1007/s00296-008-0818-y.

Lledó, A., Pastor, M. A., Pons, N., López Roig, S., Rodríguez-Marín, J., y Bruehel, S. (2010). Control beliefs, coping and emotions: Exploring relationships to explain fibromyalgia health outcomes. International Journal of Clinical and Health Psychology, 10, 459-476. Recuperado de http:/ / www.redalyc.org/articulo.oa?id=33714079005

Martin, M. Y., Bradley, L. A., Alexander, R. W., Alarcon, G. S., TrainaAlexander, M., Aaron L. A., y Alberts, K. R. (1996). Coping strategies predict disability in patients with fibromyalgia. Pain, 68, 45-53. doi: 10.1016/S0304-3959(96)03179-X

Martín-Aragón, M., Pastor, M. A., Rodríguez-Marín, J., March, M. J., Lledó, A., López-Roig, S., y Terol, M. C. (1999). Percepción de Autoeficacia en dolor crónico: Adaptación y Validación de la Chronic Pain Self-efficacy Scale. Revista de Psicología de la Salud, 11, 53-75.

Meredith, P., Strong, J., y Feeney J. A. (2006). Adult attachment, anxiety, and pain self-efficacy as predictors of pain intensity and disability. Pain, 123, 146-154. doi:10.1016/j.pain.2006.02.025

Middleton, J., Tran, Y., y Craig, A. (2007). Relationship between quality of life and self-efficacy in persons with spinal cord injuries. Arch Phys Med Rehabil, 88, 1643-1648. doi: 10.1016/j.apmr.2007.09.001)

Miró, E., Diener, F. N., Martínez, M. P., Sánchez, A. I. y Valenza, M.C (2012). La fibromialgia en hombres y mujeres: comparación de los principales síntomas clínicos. Psicothema, 1, 10-15. Recuperado de http:/ / www.redalyc.org/articulo.oa?id $=72723431002$

Pastor, M. A., Lledó, A., López, S., Pons, N., y Martín-Aragón, M. (2010). Predictores de la utilización de recursos sanitarios en la fibromialgia. Psicothema, 22, 549-555.

Pastor, M. A., Lledó, A., Pons, N., y López-Roig, S. (2011). Dolor y fibromialgia. Aportaciones desde la psicología. En C. Peñacoba (Eds.), Fi bromialgia y promoción de la salud: Herramientas de intervención psicosocial (pp 76-122). Madrid: Dykinson.

Pastor, M. A., Martín-Aragón, M., y Lledó, A. (1999). Expectativas de autoeficacia y estado de salud en enfermos con dolor crónico: una revisión. Revista de Psicología Social Aplicada, 9, 111-125.

Peters, M. L., Vlaeyen, J. W. S., y Weber, W. E. J. (2005). The joint contribution of physical pathology, pain-related fear and catastrophizing to chronic back pain disability. Pain, 113, 45-50. doi: 10.1016/i.pain.2004.09.033

Pons, N. (2009). Estado de Salud, Percepción de Control y Afrontamiento en la Fibromialgia: Un estudio longitudinal en Atención Primaria. [Tesis doctoral no publicada]. Universidad Miguel Hernández, Alicante.

Ramírez-Maestre, C., Esteve-Zarazoga, R. y López-Martínez, A. E. (2001) Neuroticismo, afrontamiento y dolor crónico. Anales de Psicología, 17, 129-137

Rivera, J., Alegre C., Ballina F., Carbonell, J., Carmona, L., Castel, B., Collado, A., Esteve, J. J., Martínez, F. G., Tornero, J., Vallejo, M. A., y Vidal, J. (2006). Documento de consenso de la Sociedad Española de Reumatología sobre la fibromialgia. Reumatología Clínica, 2, 55-66.

Rivera, J., Rejas, J., Esteve-Vives, J., y Vallejo, M. (2009). Resource utilization and health care costs in patients diagnosed with Fibromyalgia in Spain. Clinical and Experimental Rheumatology, 27, 39-45.

Ruiz-Pérez, I., Ubago M. C., Bermejo M. J., Plazaola, J., Olry De LabryLima, A., y Hernández Torres, E. (2007) Diferencias en características sociodemográficas, clínicas y psicológicas entre hombres y mujeres diagnosticados de fibromialgia. Revista clínica española, 9, 433-439

Sicras-Mainar, A., Blanca-Tamayo, M., Navarro-Artieda, R., y RejasGutiérrez, J. (2009). Costes y patrón de uso de servicios en pacientes que demandan atención por problemas mentales en asistencia primaria. Gaceta Sanitaria, 21, 306-313.

Smeets, R. J. E. M., Vlaeyen, J. W. S., Kester, A. D. M., y Kottnerus, J. A. (2005). Reduction of Pain Catastrophizing Mediates the Outcome of Both Physical and Cognitive-Behavioral Treatment in Chronic Low 
Back Pain. The Journal of Pain, 7, 261-271. doi: 10.1016/j.jpain.2005.10.011

Spinhoven, P., Kuile, M., Kole-Snijders, A., Mansfeld, M., den Ouden, D., y Vlaeyen, J. W. S. (2004). Catastrophizing and internal pain control as mediators of outcome in the multidisciplinary treatment of chronic low back pain. European Journal of Pain, 8, 211-9. doi: 10.1016/j.ejpain.2003.08.003.

Sullivan, M. J. L., Thorn, B., Haythornthwaite, J. A., Keefe, F., Martin, M., Bradley, L. A., y Lefebvre, J. C. (2001). Theoretical perspectives on the relation between catastrophizing and pain. Clinical Journal of Pain, 17, 5264.

Swinkels-Meewisse, I. E. J., Roelofs, J., Verbeek, A. L. M., Oostendorp, R. A. B., y Vlaeyen, J. W. S. (2003). Fear of movement/(re)injury, disability and participation in acute low back pain. Pain, 105, 371-379. doi:10.1016/S0304-3959(03)00255-0

Turk, D. C. (2002). A diathesis-stress model of chronic pain and disability following traumatic injury. Pain Research Management, 7, 9-19. doi: 10.2147/JPR.S38878

Turk, D. C., Okifuji, A., Sinclair, J. D., y Starz, T. W. (1996). Pain, Disability, and Physical Functioning in Subgroups of Patients with Fibromyalgia. The Journal of Rheumatology, 23, $1255-1262$

Turk, D. C., Robinson, J. P., y Burwinkle, T. (2004). Prevalence of fear of pain and activity in patients with fibromyalgia syndrome. The Journal of Pain, 5, 483-90. doi: 10.1016/j.jpain.2004.08.002
Vallejo, M. A., Comeche, M. I., Ortega, J., Rodríguez, M. F., y Díaz, M. I. (2009). Las expectativas de autoeficacia y el ajuste emocional en el afrontamiento de la fibromialgia. Escritos de Psicología, 2, 28-34.

Van Damme, S., Crombez, G., y Eccleston, C. (2002). Retarded disengagement from pain cues: the effects of pain catastrophizing and pain expectancy. Pain, 100, 111-118. doi: 10.1016/S0304-3959(02)00290-7

Velasco, L., Zautra, A., Peñacoba, C., López-López, A., y Barjola, P. (2008). Cognitive-affective assets and vulnerabilities: Two factors influencing adaptation to fibromyalgia. Psychology and Health, 21, 1-16.

Vlaeyen, J. W. S., de Jong, J., Leeuw, M., y Crombez, G. (2004). Fear reduction in chronic pain: graded exposure in vivo with behavioral experiments. In G. J. Asmundson, J. W. S. Vlaeyen and G. Crombez (Eds.), Understanding and Treating Fear of Pain. Oxford University Press, Oxford.

Vowles, K. E., McCracken, L. M., y Eccleston, C. (2007). Processes of change in treatment for chronic pain: The contributions of pain, acceptance and catastrophizing. European Journal of Pain, 11, 779-87. 10.1016/j.ejpain.2006.12.007

Wolfe, F, Smythe, H.A. Yunus, M. B, Bennett, R. M., Bombardier, C, Goldenberg, D. L., Tugwell, P., Campbell, S. M., Abeles, M., Clark, P., Fam, A. G., Farber, S. J., Fiechtner, J. J., Franklin, C. M., Gatter, R. A., Hamaty, D., Lessard, J., Lichtbroun, A. S., Masi, A. T., Mecain, G. A., Reynolds, W. J., Romano, T. J., Russell, I. J. y Sheon, R. P. (1990). The American College of Rheumatology 1990 criteria for the classification of fibromyalgia: Report of the multicenter criteria commitee. Artbritisy Rheumatism, 33(2), 160-172.

(Artículo recibido: 23-04-2012; revisado: 12-03-2013; aceptado: 13-03-2013) 